\title{
NMR Detection of Temperature-Dependent Magnetic Inhomogeneities in $\mathrm{URu}_{2} \mathrm{Si}_{2}$
}

\author{
O. O. Bernal \\ Physics Department, California State University, Los Angeles, California 90032
}

\author{
B. Becker, J. A. Mydosh, G. J. Nieuwenhuys, A. A. Menovsky, P. M. Paulus and H. B. Brom \\ Kamerlingh Onnes Lab, 2300 RA Leiden, The Netherlands \\ D.E. MacLaughlin \\ University of California, Riverside, California 92521 \\ H.G. Lukefahr \\ Whittier College, Whittier, California 90608 \\ (June 30, 1999)
}

\begin{abstract}
We present ${ }^{29} \mathrm{Si}-\mathrm{NMR}$ relaxation and spectral data in $\mathrm{URu}_{2} \mathrm{Si}_{2}$. Our echo-decay experiments detect slowly fluctuating magnetic field gradients. In addition, we find that the echo-decay shape (time dependence) varies with temperature $T$ and its rate behaves critically near $T_{\mathrm{N}}$, indicating a correlation between the gradient fluctuations and the transition to small-moment order. $T$-dependent broadening contributions become visible below $\sim 100 \mathrm{~K}$ and saturate somewhat above $T_{\mathrm{N}}$, remaining saturated at lower temperatures. Together, the line width and shift suggest partial lattice distortions below $T_{\mathrm{N}}$. We propose an intrinsic minority phase below $T_{\mathrm{N}}$ and compare our results with one of the current theoretical models.

PACS numbers: 71.27.+a, 76.60.-k, 75.10.-b, 75.25.+z, 75.30.-m
\end{abstract}

In $\mathrm{URu}_{2} \mathrm{Si}_{2}$, there is coexistence of magnetic order $\left(\mu \sim 0.04 \mu_{B} / \mathrm{U}, T_{\mathrm{N}} \sim 17.5 \mathrm{~K}\right)$ and superconductivity $\left(T_{\mathrm{c}} \sim 1.2 \mathrm{~K}\right)$ [1]. Much attention has been focused on the transition at $T_{\mathrm{N}}$ 近, with studies ultimately suggesting a quadrupolar order parameter [2]. However, no direct evidence has been found for quadrupolar ordering. $\mathrm{URu}_{2} \mathrm{Si}_{2}$ has little or no residual chemical disorder or magnetic frustration [3], but evidence for chemical order does not guarantee magnetic homogeneity [4]. Here, we report ${ }^{29} \mathrm{Si}$-NMR data (line width $\sigma$, shift $\bar{K}$, and spinecho decay rate $R$ ) which show $T$-dependent magnetic inhomogeneities below $\sim 100 \mathrm{~K}$ that correlate with the unusual magnetic order.

The sample was an oriented (alignment $\gtrsim 95 \%$ ), epoxyembedded powder (particle size $\lesssim 100 \mu \mathrm{m})$. Fig. 1(a) shows $R(T)$ obtained using conventional Hahn-echo (HE) $\left[R_{\mathrm{HE}}\right.$ : solid circles] and Carr-Purcell $(\mathrm{CP})\left[R_{\mathrm{CP}}\right.$ : open circles] sequences [5] $(H \| c$-axis $=9.4 \mathrm{~T}) . R_{\mathrm{CP}}$ is nearly $T$-independent, whereas, $R_{\mathrm{HE}}$ is not. Also, $R_{\mathrm{HE}} \gg R_{\mathrm{CP}}$ for $T \lesssim 40 \mathrm{~K}$. The shape of the HE-decay signal as a function of time $t(2 \times$ pulse-separation $)$ can be fitted to $\exp -\left(R_{H E} t\right)^{2}$ or $\exp -\left(R_{H E} t\right)^{3}$ for $T>40 \mathrm{~K}$, $\exp -\left(R_{H E} t\right)$ for $40 \mathrm{~K}>T>T_{\mathrm{N}}$, and $\exp -\left(R_{H E} t\right)^{1 / 2}$ for $T<T_{\mathrm{N}}$. $R_{H E}$ remains close to its maximum value for $T<T_{\mathrm{N}}$ with no indication of dying out at lower $T$ 's. Crude estimates of the homogeneous linewidth in the dilute limit [5] (natural abundances of ${ }^{29} \mathrm{Si},{ }^{101,99} \mathrm{Ru}: 5 \%$, $17 \%, 13 \%)$ come out too low ( $20 \%$ of $R_{\mathrm{HE}}$ at low $T$ ) and appear inconsistent with the observed $T$-dependent shape of the HE decay. Our $R$ measurements are at odds with previously reported experiments [6].
The NMR spectra was fit to a single gaussian function. $\sigma(T), K(T)$ (from the fit), and the magnetic susceptibility $\chi(T)$ (from a SQUID magnetometer) are presented in Fig. 1(b). For $T \gtrsim 100 \mathrm{~K}$, both $K$ (solid circles) and $\sigma$ (triangles) display the same $T$-dependence as $\chi$ (open circles). For $T \lesssim 100 \mathrm{~K}$, there are non-linearities in the $K$ and $\sigma$ vs. $\chi$ plots (inset). The excess width is inhomogeneous, i.e., $R_{\mathrm{HE}}$ can only account for about $1 \%$ of the total.

In a solid, there is no reason to expect a large difference between $R_{\mathrm{HE}}$ and $R_{\mathrm{CP}}$ (Fig. 1 1 (a), $T \lesssim 40 \mathrm{~K}$ ). In liquids it is quite common to find such difference, because the combination nuclear diffusion/magnetic field gradient creates more dephasing of the echo signal in the HE method; the CP method measures a smaller $R$ by eliminating the dephasing effect [5]. By comparison, having $R_{\mathrm{HE}} \gg R_{\mathrm{CP}}(T \lesssim 40 \mathrm{~K})$ here implies randomly moving or fluctuating modulations of the U-spin system that can be sensed by the static ${ }^{29} \mathrm{Si}$-nuclei. We believe that this is the first time that such effect is measured in a heavyfermion system.

The $T$-dependence of $R_{\mathrm{HE}}$ and $\sigma$ could be suggesting local charge-density wave (CDW) structures as a source of U-spin modulations. In CDW systems, $R$ can display a $\left(T-T_{0}\right)^{-1 / 2}$ dependence for $T \gtrsim T_{0}\left(T_{0}\right.$ : CDW transition temperature) [7] [inset Fig. [1(a); $T_{0} \rightleftharpoons T_{N}$ ]. Also, incommensuration/randomness of forming CDW's would distribute the $\mathrm{U}^{29} \mathrm{Si}$ transferred hyperfine coupling locally and explain the observed $T$-dependence of $\sigma$. In addition, there is no clear change in NMR line shape below $T_{\mathrm{N}}$, so only a fraction of the ${ }^{29} \mathrm{Si}$ nuclei must be 
affected; the majority would sample an underlying paramagnetic fluid. Given the coexistence of magnetism and superconductivity [1] and that two kinds of transitions seem to be needed to explain the macroscopic anomalies [2], an interpretation in terms of two different phases (as opposed to introducing a $T$-dependent hyperfine coupling) seems plausible. Therefore, we fit the data using the simple relations $K=K_{0}+a \chi(T)+K_{\alpha}(T)$, and $\sigma^{2}=\sigma_{0}^{2}+(\delta a)^{2} \chi^{2}(T)+\sigma_{\alpha}^{2}(T)$. Here $K_{0}=0.06 \%$ (contact hyperfine interaction), $a=3.5 \mathrm{kOe} / \mu_{B}, \sigma_{0}=0.005 \%$, and $(\delta a) \sim 0.5 \mathrm{kOe} / \mu_{B} . K_{\alpha}(T)$ and $\sigma_{\alpha}(T)$ are the contributions due to the second phase.

In Fig. 11(c), a plot of $\sigma_{\alpha}$ vs. $K_{\alpha}$ shows proportionality at high $T$ (slope -1$)$ and a breakdown of linearity somewhat above $T_{\mathrm{N}}$. This is more qualitative evidence that the inhomogeneous magnetism is correlated with the unusual transition. The slope in this plot represents the fractional width of the distribution of U- ${ }^{29} \mathrm{Si}$ transferred hyperfine couplings for the second phase. The abrupt slope change just above $T_{\mathrm{N}}$ (from $\sim-1$ to $\sim 0$ ) would signal an intrinsic rearrangement of the U-spins in this phase with respect to the ${ }^{29} \mathrm{Si}$ nuclei, i.e., partial lattice distortions, since no distortion affecting the whole crystal has been detected (see [1,2]). The saturation of $\sigma_{\alpha}$ below $T_{\mathrm{N}}$ indicates that these distortions remain at lower $T$.

In conclusion, we have found evidence for random or incommensurate structures in $\mathrm{URu}_{2} \mathrm{Si}_{2}$ at low $T$. We propose slowly moving and/or fluctuating charge-density modulations, which could in turn serve as domain walls to antiferromagnetic regions consistent with the resistance anomaly measured at $T_{\mathrm{N}}$ [1]. One could also argue that our results (local distortions near, but not at, $T_{\mathrm{N}}$, as well as U-spin modulation fluctuations when $T \rightarrow T_{\mathrm{N}}$ ) are consistent with the picture presented in Ref. [2]. Finally, the spectra for $H \perp c$-axis are also consistent with $\mathrm{U}$-spins modulations [2], Fig. 11(d). Systematic field and orientation dependence studies of the NMR anomaly are being carried out to make progress in this direction.

We acknowledge helpful discussions with R.E. Walstedt and D.L. Cox. This research was supported by an award from Research Corporation and NSF grant DMR9820631 .
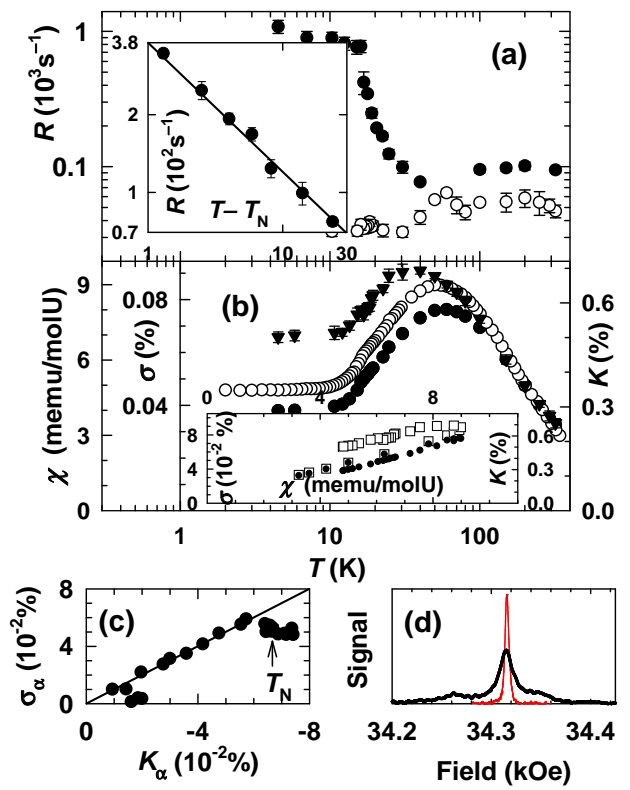

FIG. 1. (a) $R$ vs. $T(H \| c$-axis=9.4 T); circles: HE (solid), $\mathrm{CP}$ (open). Inset: $R_{\mathrm{HE}}$ vs. $T-T_{\mathrm{N}} ; T_{\mathrm{N}} \sim 17 \mathrm{~K}$ (line: fit to $\left.A T_{\mathrm{N}}^{1 / 2} /\left(T-T_{\mathrm{N}}\right)^{1 / 2} ; A \sim 123 \mathrm{~s}^{-1}\right)$. (b) Circles: $\chi$ (open) and $K$ (solid) vs. $T$. Triangles: $\sigma$ vs. $T$. Inset: $\sigma$ (squares) and $K$ (circles) vs. $\chi, T$ implicit. (c) $\sigma_{\alpha}(T)$ vs. $K_{\alpha}(T)$. (d) Spectra ( $H \perp c$-axis): $T=25 \mathrm{~K}$ (narrower line), $4.2 \mathrm{~K}$ (broader line).

[1] T.T.M. Palstra et al., Phys. Rev. Lett. 55 (1985) 2727; S.A.M. Mentink et al., Phys. rev. B 53 (1996) R6014; G.J. Nieuwenhuys, Phys. Rev. B 35 (1987) 5260; V. Barzykin and L.P. Gor'kov, Phys. Rev. Lett. 74 (1995) 4301; P. Santini and G. Amoretti, Phys. Rev. Lett. 73 (1995) 1027; K. Kuwahara et al., J. Phys. Soc. Japan 66 (1997) 3251; J.G. Rodrigo et al., Phys. Rev. B 55 (1997) 14318; and references therein.

[2] P. Santini, Phys. Rev. B 57 (1998) 5191.

[3] J.A. Mydosh, Z. Phys. 103 (1997) 251.

[4] O.O. Bernal et al., Phys. Rev. Lett. 75 (1995) 2023; D.E. MacLaughlin et al., Phys. Rev. B 58 (1998) R11849.

[5] C.P. Slichter, Principles of Magnetic Resonance, 3rd edition (Springer, NY, 1990) p. 367.

[6] Y. Kohori et al., J. Phys. Soc. Japan 65 (1996) 1083.

[7] C. Berthier, D. Jerome and P. Molinie, J. Phys. C: Solid State Phys. 11 (1978) 797. 